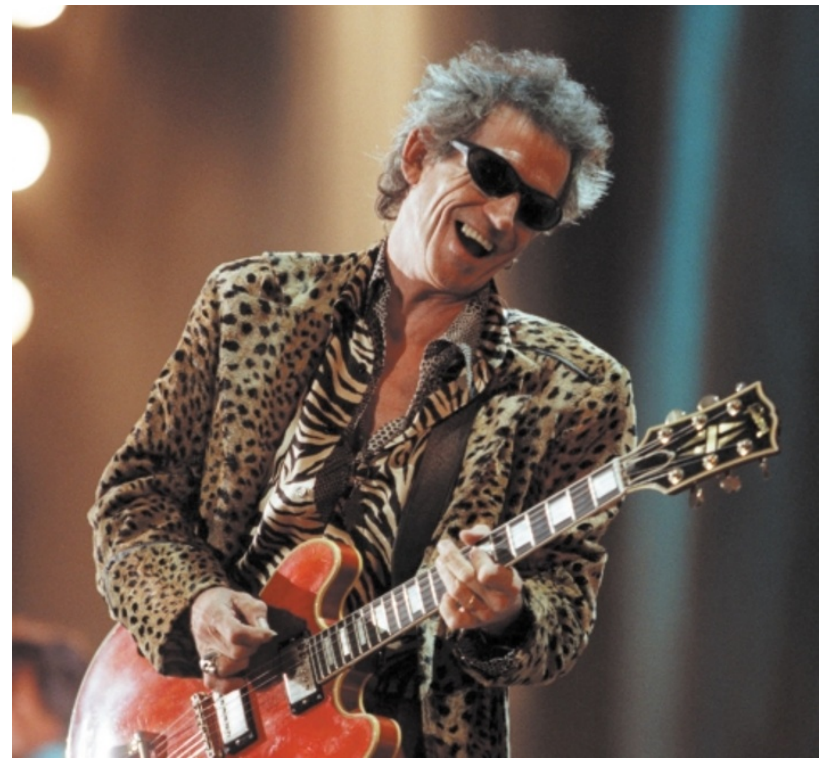

(c) Associated Press.

MOVEMENT DISORDERS

\section{Re-tuning the system}

For rock musicians, an episode of cramp may well result in a wrong note, but it is unlikely to result in headlines across the world in quite the same way as some of the other 'occupational' hazards of being a rock star. Musician's cramp is nonetheless a serious problem for those afflicted and is part of a wider class of disorders known as task-specific focal dystonias, which are characterized by involuntary movements and abnormal and often painful postures. Dystonia is often refractory to pharmacological intervention and the underlying mechanisms are unclear at present.

Monkey studies indicate that sensory cortical representations might be disrupted in focal dystonias. However, it is uncertain whether these changes are the cause or the result of the motor abnormalities observed in dystonia. Given the plasticity of these systems, abnormal postures might be expected to lead to changes in sensory systems over time, but it is less clear how motor changes could be triggered by sensory modification. Sanger and Merzenich have addressed this problem by devising a computational model to explain how modifications to sensory representations could lead to abnormal motor behaviour with the characteristics of focal dystonia. The idea is that dystonia might result from an increased gain in the sensorimotor control loop. As gain is determined in part by the size of the sensory cortex devoted to a particular movement, behaviours that lead to abnormal increases in sensory cortical representations are predicted to lead to dystonia. Simulations showed that several different mechanisms that increased gain via plasticity within the sensory cortical representation led to sustained and uncontrolled motor activity in the sensorimotor loop. Although the clinical relevance of this model remains to be tested, the demonstration that changes in sensory representation could provide a mechanism for the aetiology of dystonia represents an intriguing development. Indeed, re-tuning the sensory representation of patients with dystonia might even be a possible treatment strategy.

Peter Collins

(2) References and links

ORIGINAL RESEARCH PAPER Sanger, T. D. \& Merzenich, M. M. Computational model of the role of sensory disorganization in focal task-specific dystonia. J. Neurophysiol. $\mathbf{8 4}$, 2458-2464 (2000)

FURTHER READING Hallet, M. The neurophysiology of dystonia. Arch. Neurol. 55 601-603 (1998)

ENCYCLOPEDIA OF LIFE SCIENCES Somatosensory systems

WEB SITES Michael Merzenich | The Dystonia Society

H I G H L I G H T S

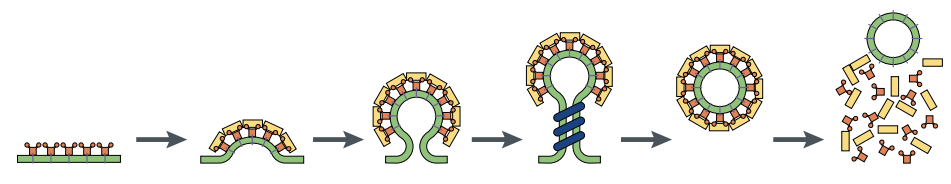

NEUROTRANSMITTER RECEPTORS

\title{
AMPA receptors: now you see them, now you don't
}

It has been an intense month for the study of AMPA-receptor endocytosis. Although the idea that endocytosis can regulate the availability of this receptor subtype was controversial for some time, the evidence in favour of this hypothesis is now stronger. Later experiments have therefore moved on to explore the possible cellular mechanisms that control the internalization of AMPA receptors. Three recent papers in Neuron and Nature Neuroscience attest to the rapid development of this field and, at the same time, begin to show us that it will not be easy to obtain a definitive mechanistic explanation.

Michael Ehlers found that AMPAreceptor endocytosis occurs through two separate pathways in cultured neurons, depending on the nature of the stimulus that triggers internalization; NMDA application was accompanied by fast AMPA-receptor endocytosis and recycling back to the synaptic membrane, whereas AMPA application targeted receptors to lysosomal degradation. In addition, receptor trafficking induced by NMDA, but not by AMPA, was regulated by the opposing activities of protein kinase A on the one hand, and protein phosphatase-1 (PP-1) and calcineurin on the other.

Beattie and colleagues obtained similar results regarding the effect of NMDA application. Although they did not investigate the fate of the internalized receptors, they showed that AMPA-receptor endocytosis elicited by NMDA application was accompanied by a decrease in the frequency of spontaneous synaptic events. In addition, NMDA-induced AMPA-receptor endocytosis occured preferentially in dendrites, whereas the effects of AMPA application were primarily observed on somatic recep- tors. However, Beattie et al. found that the effect of AMPA was also affected by the application of calcineurin antagonists, an observation opposite to that made by Ehlers.

Lin and colleagues, in turn, agreed in this respect with Ehlers, as they did not observe an effect of PP- 1 or calcineurin antagonists on the AMPAinduced receptor endocytosis. However, Lin et al., who did not investigate the effect of NMDA application, failed to obtain evidence that AMPAinduced internalization elicited lysosomal targeting of the receptors and proposed that AMPA receptors quickly reappear in the cell surface after AMPA application.

The differences among these studies are intriguing and could perhaps be resolved by studying the effect of synaptically released glutamate, instead of the exogenous application of agonists. However, the fact that AMPA receptors can be internalized through different pathways depending on the nature of the stimulus implies a far more complex receptor regulation than previously thought. This prompts new questions about the role of these pathways in the plastic changes that occur at the synapse. Juan Carlos López

(2) References and links ORIGINAL RESEARCH PAPERS Ehlers, M. D. Reinsertion or degradation of AMPA receptors determined by activity-dependent endocytic sorting. Neuron 28, 511-525 (2000) | Lin, J. W. et al. Distinct molecular mechanisms and divergent endocytic pathways of AMPA receptor internalization. Nature Neurosci. 3, 1282-1290 (2000) | Beattie, E. C. et al. Regulation of AMPA receptor endocytosis by a signaling mechanism shared with LTD. Nature Neurosci. 3, 1291-1300 (2000) FURTHER READING Haucke, V. Dissecting the ins and outs of excitement: glutamate receptors on the move. Nature Neurosci. 3, 1230-1232 (2000) | Slepnev, V. I. \& De Camilli, P. Accessory factors in clathrin-dependent synaptic vesicle endocytosis. Nature Rev. Neurosci. 1, 161-172 (2000) | Scannevin, R. H. \& Huganir, R. L. Postsynaptic Scannevin, R. H. \& Huganir, R. L. Postsynaptic
organization and regulation of excitatory synapses. Nature Rev. Neurosci. 1, 133-141 (2000) 